\title{
LA ENSEÑANZA SITUADA PARA EL DESARROLLO DE COMPETENCIAS EN LOS ESTUDIANTES A TRAVÉS DE PRÁCTICAS GUIADAS
}

\author{
SITUATED TEACHING FOR THE DEVELOPMENT OF SKILLS IN STUDENTS \\ THROUGH GUIDED PRACTICES
}

Recibido: 24 de septiembre 2021

Aceptado: 6 de noviembre de 2021
K. Berlanga Reséndiz ${ }^{1}$ S.E. Barrios Mendoza ${ }^{2}$ R. Altamirano Zúñiga ${ }^{3}$ M. V. Molina Cantú ${ }^{4}$

\section{RESUMEN}

El presente trabajo demuestra el impacto del diseño de un manual de prácticas como material didáctico complementario para desarrollo de las competencias específicas de la materia Calidad Aplicada a la Gestión; a través del mismo es posible comprobar que al desarrollar actividades prácticas adecuadas, los estudiantes son capaces de aplicar sus conocimientos para proponer soluciones pertinentes a problemas específicos, en este caso se desarrolló un proyecto en una empresa dedicada a la construcción y montajes industriales, que consistía en la identificación y análisis de las causas que generan los productos defectuosos en el área de pintura, se determinó los principales defectos que se presentan en el área de pintura; falta de adherencia, polvo y suciedad, falta de cubrición, marcas de lijado, arrugados, corrosión interna, defecto de curado, manchas de agua. Detectándose como las más comunes piel de naranja o falta de nivelación, hervidos o burbujas de disolvente, descuelgues y falta de limpieza en la superficie. Con el análisis de las causas que generaban estos defectos se identificaron las causas principales, con lo que fue posible elaborar una propuesta de un plan de acción que permitirá a la empresa mejorar y disminuir los productos defectuosos evitando reproceso y optimizando los recursos.

PALABRAS CLAVE: Vinculación Empresa-escuela, Calidad, Competencias Profesionales.

\section{ABSTRACT}

The present work demonstrates the impact of the design of a practice manual as a complementary didactic material for the development of the specific competences of the subject Quality Applied to Management, it is possible to verify, in the students of Engineering in Business Management, how by developing appropriate practical activities, they are able to apply their knowledge to propose solutions, in this specific case a project was developed in a company dedicated to construction and industrial assemblies, which consisted in the Identification and Analysis of the causes that generate defective products in the painting area, it was determined the main defects that occur in the painting area; lack of adherence, dust and dirt, lack of coverage, sanding marks, wrinkles, internal corrosion, curing defects, water stains. The most common causes are orange peel or lack of leveling, boiling or solvent bubbles, sagging and lack of surface cleanliness. With the analysis of the causes that generated these defects, the main causes were identified, with which it was possible to develop a proposal for an action plan that will allow the company to improve and reduce defective products, avoiding reprocessing and optimizing resources.

KEY WORDS: Collaboration, School company, Quality, Professional skills.

\footnotetext{
${ }^{1}$ Docente del Tecnológico Nacional de México Campus Ciudad Valles, karina.berlanga@tecvalles.mx

${ }^{2}$ Docente del Tecnológico Nacional de México Campus Ciudad Valles, silvia.barrios@ tecvalles.mx

${ }^{3}$ Docente del Tecnológico Nacional de México Campus Ciudad Valles, raul.altamirano@tecvalles.mx

${ }^{4}$ Docente del Tecnológico Nacional de México Campus Ciudad Valles victoria.molina@tecvalles.mx
} 


\section{INTRODUCCIÓN}

De acuerdo con el Modelo Educativo para el Siglo XXI; Formación y desarrollo de competencias profesionales en su Dimensión académica menciona que: "La construcción de competencias implica establecer modelos de movilización de conocimientos de manera adecuada, en tiempo real y al servicio de una acción eficaz" (Acosta González \& al, 2012). También indica que, para crear estos modelos, se debe analizar e integrar las experiencias derivadas de la práctica profesional, que renueva y estructura estas herramientas didácticas. Por eso, es lógico que las instituciones educativas, y la sociedad en su conjunto, se vinculen de manera estrecha y corresponsable para que, ante la cambiante, compleja e impredecible situación global, marcada por constantes fenómenos emergentes, se configuren prácticas profesionales y culturales que favorezcan la búsqueda de nuevas tendencias de formación profesional, nuevos modelos de movilización de conocimientos, especialmente en el campo de las competencias, como el camino más fiable para orientar los procesos educativos en una dirección que armonice las necesidades individuales y sociales, en la inteligencia de que la acción educativa-formativa va más allá del ámbito escolar, toda vez que se refleja e influye en el desarrollo de la sociedad misma." (Acosta González \& al, 2012)

En este sentido el docente se concibe como un mediador que debe incorporar recursos y estrategias didácticas que permitan influir en forma positiva en el desarrollo proceso de aprendizaje, lo que permitirá la construcción de competencias específicas que faciliten al estudiante una adaptación al contexto laboral y a la vez desarrollar confianza en sí mismo. (Maldonado Pérez, 2008) El docente debe ser capaz de diseñar estrategias didácticas y generar ambientes de aprendizaje que faciliten el logro de aprendizajes esperados en los planes de estudio y que permitan lograr una vinculación benéfica entre docente-contenidorealidad-estudiante. (Maldonado Pérez, 2008)

El desarrollo de estrategias didácticas busca que los estudiantes vayan más allá que la simple memorización de conceptos o teorías, sino que también sean capaces de analizar e interpretar al mundo que los rodea de una manera crítica y poder plantear soluciones y mejoras de forma responsable ante situaciones específicas. El aprendizaje basado en proyectos establece el propósito de guiar al conocimiento de una forma estructurada, orientada y formativa, esto es todavía mejor cuando se realiza a través de la vinculación escuela-empresa, esto para que el estudiante pueda percibir de forma clara y real al entorno laboral actual. (Fajardo Pascagaza, 2019)

La enseñanza situada son actividades para integrar a los estudiantes con la comunidad a través de prácticas sociales. Es por ello por lo que Díaz Barriga (2003) menciona lo siguiente: "Para la enseñanza situada aprender y hacer son acciones inseparables, por lo que los alumnos han de aprender haciendo dentro del contexto pertinente", él explica que el conocimiento adquirido en la educación "in situs" es una de las tendencias más distintivas dentro del enfoque sociocultural.

El aprendizaje a través de prácticas guiadas va de la mano con el basado en proyectos el cual promueve que el estudiante sea capaz de planificar el trabajo, escuchar, negociar y tomar decisiones; para poder evaluar en conjunto con su equipo o guías asesores y plantear soluciones e innovaciones. (Cobo Gonzales \& Valdivia Cañotte, 2017) En otro estudio se analiza la efectividad de realizar la vinculación escuela-empresa a través de proyectos, permite integrar conocimientos adquiridos durante su formación profesional al poner en 
práctica estos en el campo laboral, también indican se aumenta la facilidad para la toma de decisiones con fundamento, y esto les permite a los estudiantes brindar aportaciones en áreas específicas de la empresa. Con la realización de proyectos propicia el trabajo en equipo además de brindarles sentido de responsabilidad; al integrar conocimientos y habilidades de cooperación y colaboración entre los participantes, y mejora la habilidad de comunicación de los estudiantes. (Maldonado Pérez, 2008). Con el aprendizaje basado en proyectos en la empresa, el estudiante tiene la oportunidad de formarse en un escenario real, establecer relaciones a través de la interacción con ejecutivos, supervisores y trabajadores reales. (Ferreiro Martínez, Brito Laredo, Isabel Garambullo, \& Martínez López, 2012) Es necesario reiterar la importancia que tiene el docente como guía en los aprendizajes deseados. El aprendizaje basado en problemas (ABP) consiste en el planteamiento de una situación problema, en este caso en cuestión a través de prácticas en un contexto real donde su construcción, análisis y solución constituyen no solo aprendizaje sino también experiencias reales para los estudiantes. (Díaz Barriga Arceo, 2006)

El trabajo que se presenta a continuación muestra como a través del desarrollo de prácticas guiadas en la materia de "Calidad Aplicada a la Gestión" desarrolladas directamente en una empresa fue posible tipificar las causas que generaban los principales defectos que se presentan en el área de pintura; en el estudio se hizo uso de las herramientas de la calidad y el estudiante demostró sus habilidades y conocimientos para poder diseñar estrategias que permitieran a la empresa disminuir los productos defectuosos, evitar reprocesos y optimizar recursos.

\section{METODOLOGÍA}

\section{Diseño de la Investigación}

Se realiza una investigación causal explicativa, en la cual se llevaron a cabo distintas actividades para garantizar una adecuada identificación de la oportunidad de mejora en donde se logrará la aplicación de herramientas estadísticas que permitan identificar los tipos de defectos en el área de pintura.

1. Identificación del problema en la empresa, como primera fase se realizó un diagnóstico. La necesidad de realizar un diagnóstico se centra en la importancia de que necesario conocer la situación y el contexto para poder tomar las acciones correctas. Durante varios recorridos por la empresa y la aplicación de entrevistas con personal clave de esta se logró identificar el área que en el momento de estudio estaba generando una serie de situaciones de crisis en la empresa, se identificó "El área de pintura" como un área con un notable incremento de las "no conformidades ", esto debido a los defectos que se producían constantemente.

Debido a lo anterior se determinó que era necesaria la aplicación de herramientas estadísticas que permitieran identificar los tipos de defectos y análisis de las causas que generaban las no conformidades registradas con el fin de obtener soluciones a corto y mediano plazo.

2. Se utilizó una lista de verificación para recopilar la información acerca del tipo de defectos que se presentaban

3. Se diseñó una plantilla en Excel para concentrar y analizar la información obtenida

4. Durante un periodo de 2 meses se realizó la recolección de información

5. Se aplicaron herramientas estadísticas para identificar las causas de los defectos. 


\section{RESULTADOS}

Los defectos se clasificaron especificando fecha y el proyecto en el que se presentaban. La piel de naranja o falta de nivelación es un tipo de defecto que se presentó en 3 diferentes proyectos los cuales son:FMI_20067, FMI_20069 y FMI_20005, (ver gráfica 1), este defecto consiste en que la superficie se encuentra mal igualada por falta de estiramiento o nivelación. Algunas de las causas pueden ser: Disolventes demasiados rápidos o capaz de fondo poco secas, exceso de producto por pasada de pistola o bien distancia inadecuada de aplicación

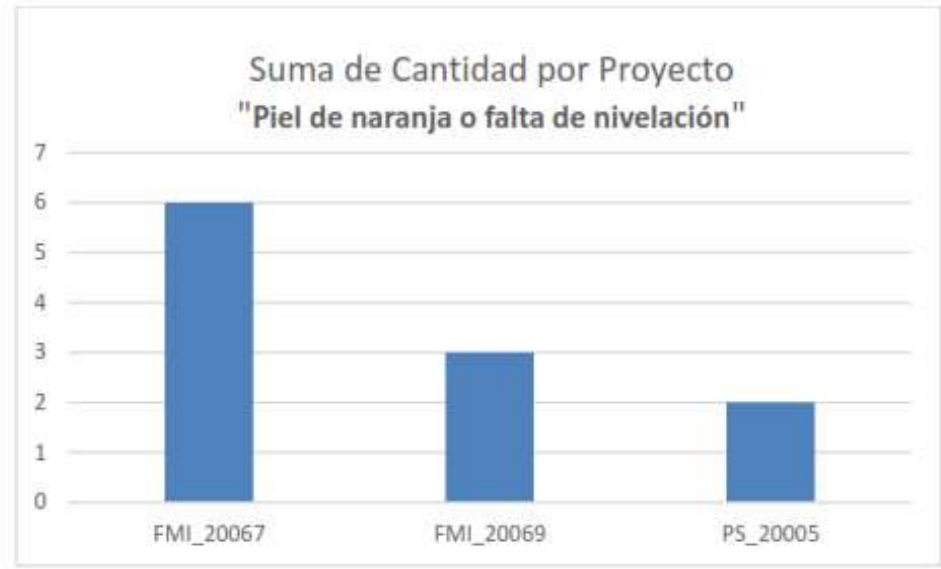

Gráfica 1. Defectos presentados: Piel de naranja o falta de nivelación

Otro de los defectos más frecuentes fue "hervidos" o burbujas disolventes, pequeños picados superficiales producidos por rotura o deformación de la superficie de pintura, generados por la salida de disolventes ocluido una vez seca la superficie exterior de la capa de pintura. El cual se presentó en 5 proyectos los cuales se pueden observar en el diagrama de barras. (Ver gráfica 2). Las causas más frecuentes de este defecto son: Disolventes demasiados rápidos, temperatura ambiente alta, capas muy gruesas y polvo ambiental.

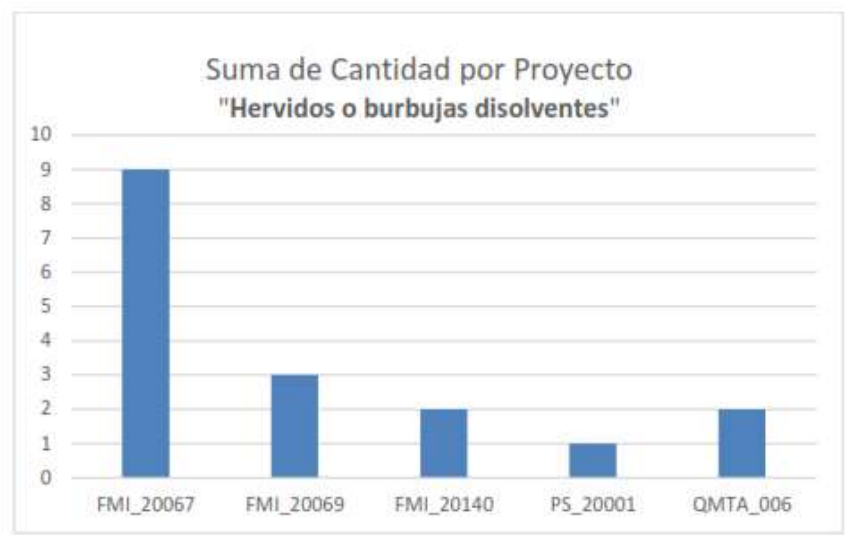

Gráfica 2. Defectos presentados: hervidos o burbujas disolventes

La presencia de óxido se hace ver debido a la humedad, desencadenando la reacción electroquímica del metal con el oxígeno apareciendo ampollas o decoloraciones en la superficie de la pintura, la causa principal de este defecto cuando ya la superficie fue pintada es la exposición ambiental o bien que no se realizó una limpieza profunda como se debía. 
FMI_20069 fue el único proyecto que en 4 ocasiones presento este desperfecto (Ver gráfica 3).

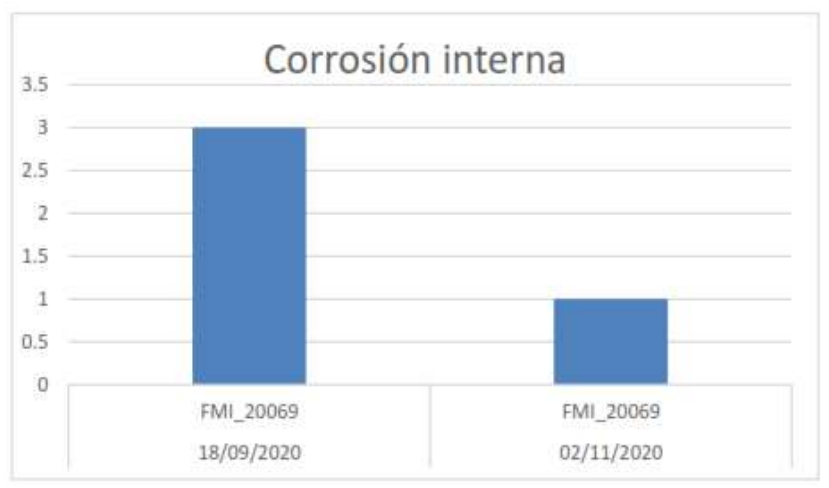

\section{Gráfica 3. Defectos presentados: corrosión interna.}

Cuando la pintura tiene una baja viscosidad debido a un exceso de disolvente o una inadecuada regulación de la aplicación (baja presión del aire, excesivo caudal de producto y defecto de distancia de aplicación). Produce escurridos en la pintura.

Los descuelgues estuvieron presente en diversas ocasiones y en un promedio de 5 proyectos, algunos de los métodos para solucionar este defecto podría ser, el aplicar varias capas finas, atemperar ligeramente la pintura o bien ajustar y regular el equipo para evitar la baja precisión del aire. (Ver gráfica 4).

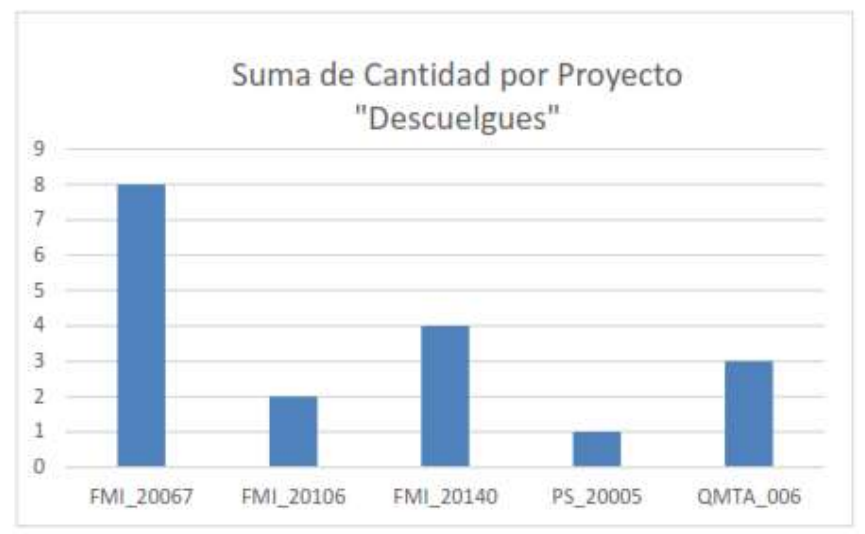

\section{Gráfica 4. Defectos presentados: Descuelgues con escurrimiento de pintura}

El defecto de arrugados es uno de los defectos menos frecuentes en esta industria y consiste en pequeños pliegues superficiales producidos durante la aplicación o su secado, este defecto solo se presentó por 3 ocasiones en el proyecto FMI_20067, la causa de dicho defecto fue la reacción sobre capas de fondo incompatibles y la utilización de disolvente inadecuado. (Ver gráfica 5). 


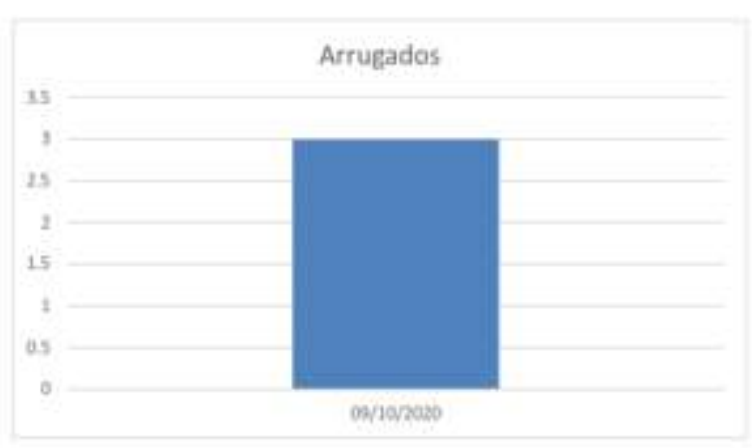

\section{Gráfica 5. Defectos presentados: Arrugado con pequeños pliegues}

En diversas ocasiones el material una vez que lo recubrieron necesita ser trasladado a otro lugar para despejar el área de trabajo y sufre un despintado pues no se espera el tiempo adecuado y esto proporciona marcas, la manera en que se pudiera evitar es respetando los tiempos de secado que indican las fichas técnicas de las pinturas o bien adicionando acelerante siempre y cuando el cliente lo autorice. No es un defecto muy frecuente sin embargo se presentó en 2 proyectos. (Ver gráfica 6).

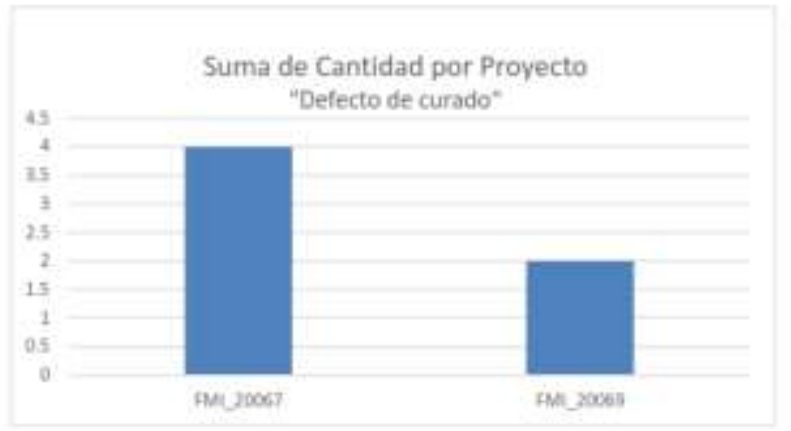

\section{Gráfica 6. Defectos presentados: Defecto de curado}

Cuando la pintura aun no cumple con el tiempo de secado y tiene un contacto con el agua u algo que tenga humedad crea cercos blanquecinos. Las causas más frecuentes es la exposición a un ambiente húmedo del soporte de pintado y salpicaduras de agua o gotas de agua en la superficie pintada. Esto se pudiera evitar respetando las condiciones del secado y trabajar dentro de un área adecuada para evitar el contacto con la humedad. (Ver gráfica 7).

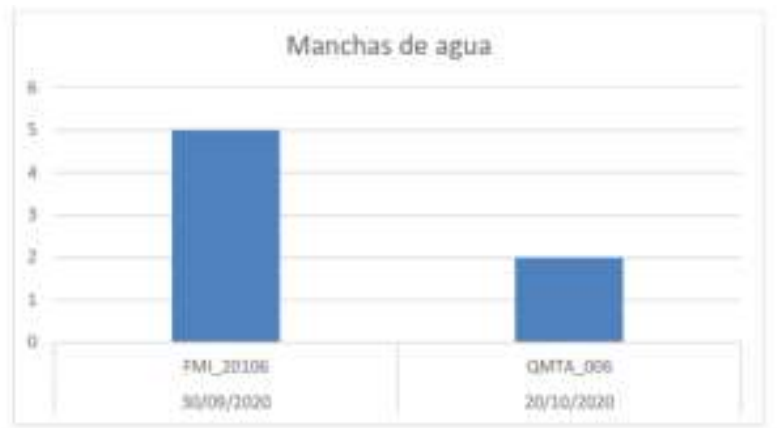

Gráfica 7. Defectos presentados: Manchas de agua 
En diversas ocasiones la lija es un instrumento que se utiliza para el desarrollo de la limpieza de superficie y cuando ese lijado no es cubierto o rellenado adecuadamente por la pintura son producidos rayas o surcos los cuales definen al defecto "Marcas de lijado".

Después de un primer recubrimiento es realizado un lijado para eliminar los pequeños detalles que pudiera tener cada ensamble y una de las causas que lo producen es la falta de secado o endurecimiento de la superficie o una elección inadecuada del grosor de la lija. Para evitar que el defecto se haga presente es conveniente seleccionar adecuadamente cada instrumento y esperar el tiempo adecuado. Este defecto es uno de los menos comunes dentro de JRO estuvo presente en el proyecto FMI_20067 en tres diferentes ocasiones. (Ver gráfica $8)$.

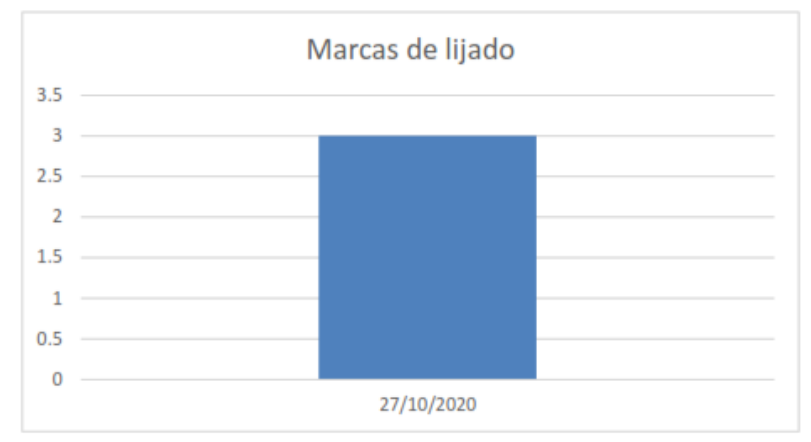

\section{Gráfica 8. Defectos presentados: Marcas de lijado}

Este defecto es muy explícito su nombre lo dice, es la presencia de contaminación por polvo y suciedad sobre la superficie pintada, algunas de las causas más comunes son; lugar de pintado deficiente o en malas condiciones o la presencia de contaminantes en la pintura. Este defecto se encontró en 2 ocasiones en el proyecto FMI_20067. (Ver gráfica 9).

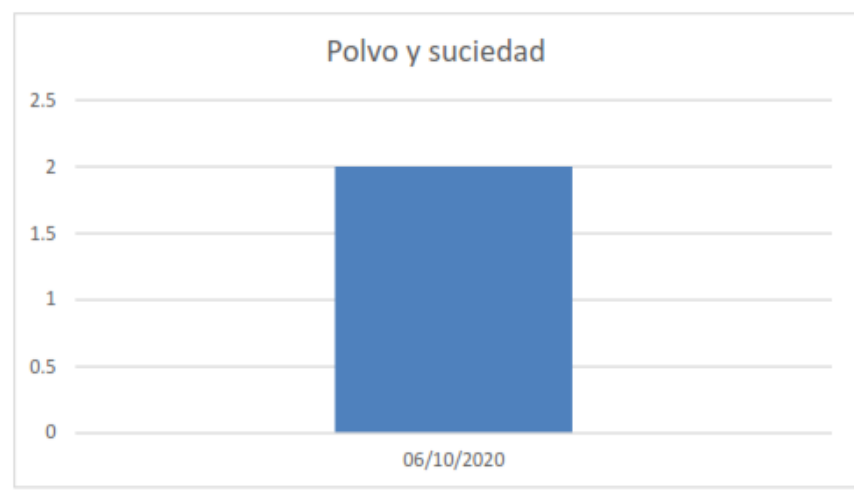

Gráfica 9. Defectos presentados: Polvo y suciedad

Cuando la pintura ya seca se desprende fácilmente es a causa de una inadecuada preparación de la superficie, fondos en mal estado o un lijado deficiente, en el proyecto FMI_20067 se presentó en 2 ocasiones. (Ver gráfica 10). 


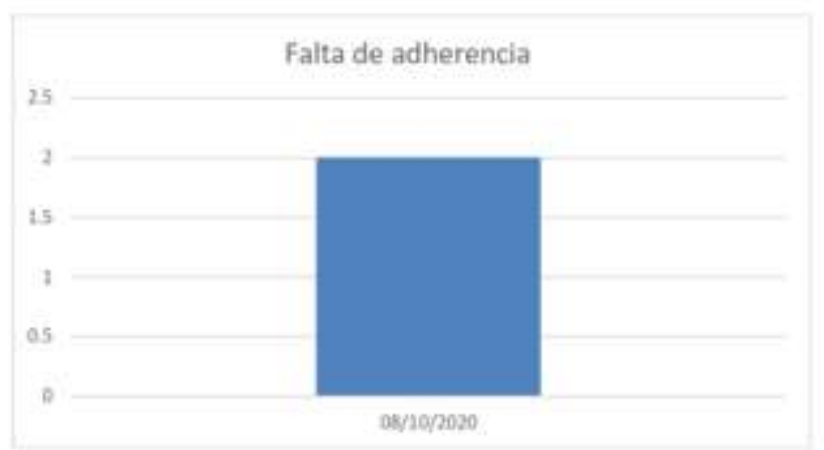

\section{Gráfica 10. Defectos presentados: Falta de adherencia}

Son 4 las limpiezas de superficie más utilizadas dentro de la empresa para lograr un adecuado recubrimiento, las normas SSPC-SP son las bases de los procedimientos para que estas se desempeñen de manera adecuada, sin embargo, es uno de los defectos más frecuentes fue detectado en cerca de 20 ocasiones. (Ver gráfica 11).

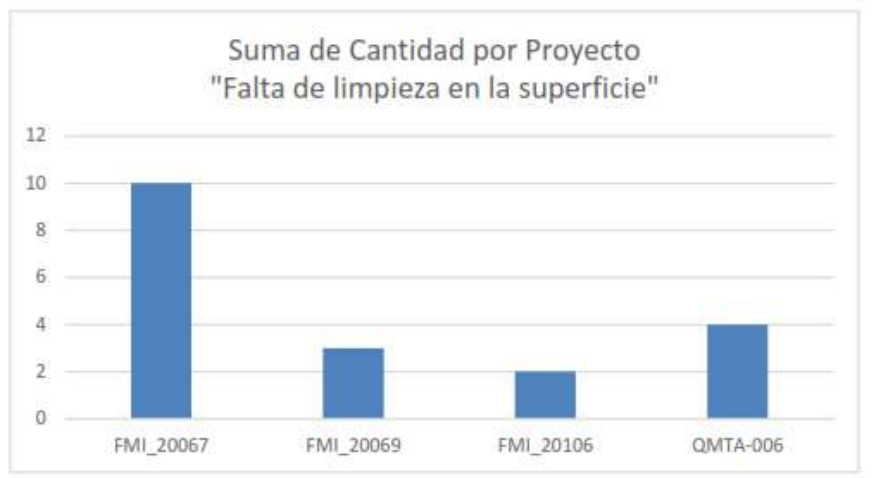

Gráfica 11. Defectos presentados: Falta limpieza en la superficie

Aplicando el Histograma y utilizando las bases del Diagrama de Pareto que dice que el $20 \%$ de las causas provoca el $80 \%$ de los problemas en defectos se obtiene el siguiente gráfico. (Ver gráfica 12).

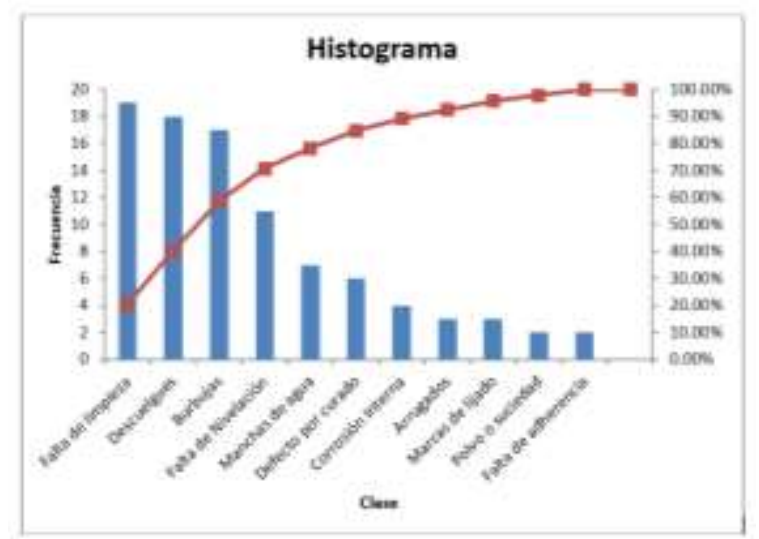

Gráfica 12. Histograma de defectos presentados 
Por lo que se identifica que el mayor número de no conformidades lo causan:

1. Falta de limpieza

2. Descuelgues

3. Burbujas

4. Falta de Nivelación

Con la información identificada en la actividad anterior y con la participación del personal involucrado se desarrolló un diagrama Ishikawa de causa- efecto (Saeger, 2016), con el fin de identificar cuáles son las causas potenciales que generan los defectos identificados. (Ver esquema 1).

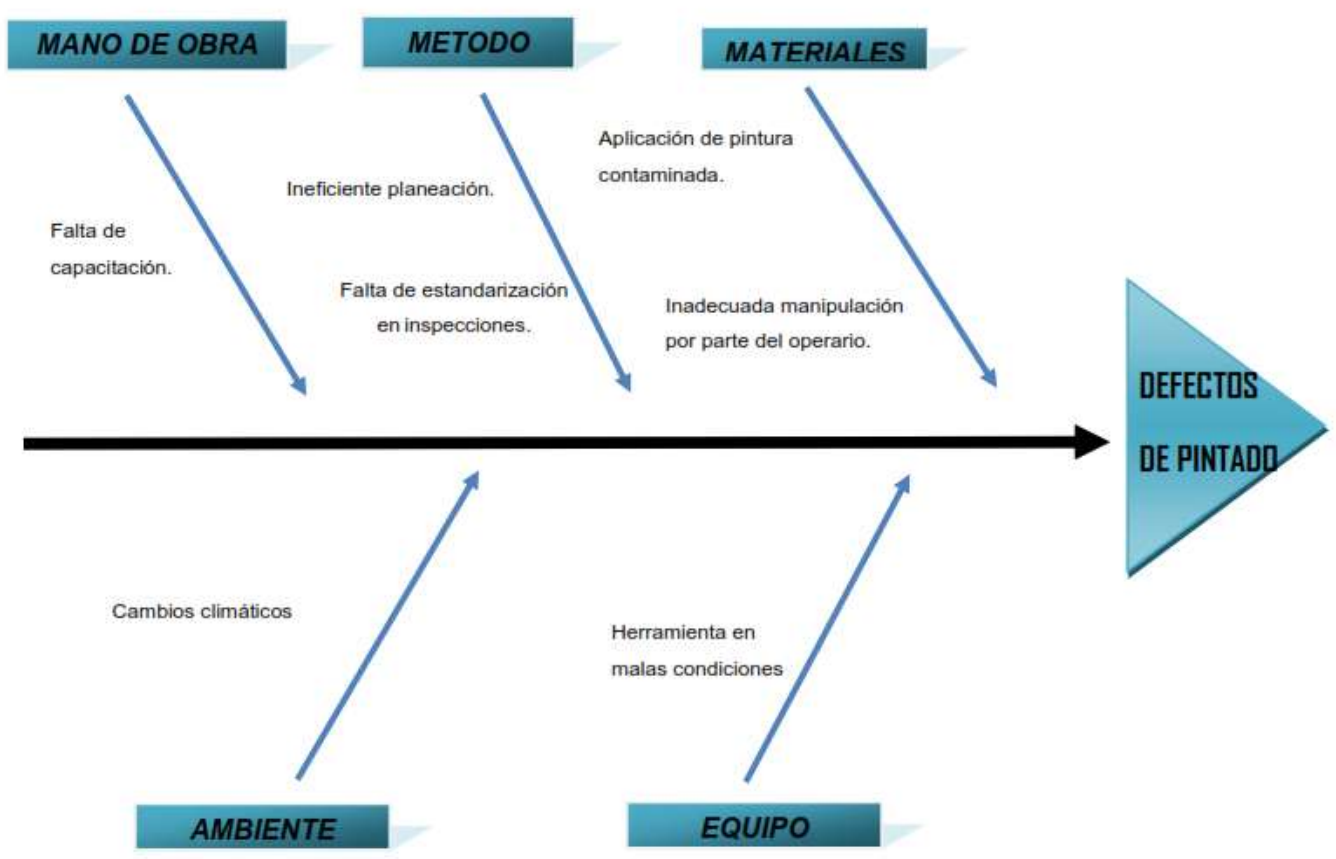

Figura 1. Diagrama de Ishikawa de las causas potenciales que originan los defectos. Fuente propia.

Utilizando la técnica multivoting se identifica que de las causas principales son:

a) Falta de capacitación del personal que participa en el proceso de pintado

b) Falta de planeación de las actividades a realizar

c) Falta de adecuación del área de trabajo para el tipo de proceso que se lleva a cabo.

\section{Propuesta del Plan de acción}

Problema: Incremento de no conformidades en el área de pintura

\section{Principales defectos encontrados}

1. Falta de limpieza

2. Descuelgues

3. Burbujas

4. Falta de Nivelación 


\section{Causas identificadas:}

a) Falta de capacitación del personal que participa en el proceso de pintado

b) Falta de planeación de las actividades a realizar

c) Falta de adecuación del área de trabajo para el tipo de proceso que se lleva a cabo.

\begin{tabular}{|l|l|l|}
\hline Numero & Actividad & Responsable \\
\hline 1 & $\begin{array}{l}\text { Elaborar procedimientos e instructivos de trabajo con } \\
\text { las actividades adecuadas para las actividades } \\
\text { desarrolladas en el área de pintura. }\end{array}$ & Jefe de área de pintura \\
\hline 2 & $\begin{array}{l}\text { Elaborar un catálogo con los tipos de defectos que } \\
\text { puedan presentarse y las actividades a realizar para. }\end{array}$ & Jefe de área de pintura \\
\hline 1. Evitarlos & $\begin{array}{l}\text { En caso de que sea imposible evitarlos, } \\
\text { corregirlos. }\end{array}$ & \\
\hline 3 & $\begin{array}{l}\text { Capacitar al personal que desarrolla el proceso para } \\
\text { concientizarlos de la importancia de su trabajo en la } \\
\text { satisfacción del cliente }\end{array}$ & Jefe de área de pintura \\
\hline 4 & $\begin{array}{l}\text { Realizar un estudio para elaborar una propuesta de } \\
\text { adecuación del área de trabajo en cuanto a } \\
\text { condiciones físicas, infraestructura, espacio, etc. con } \\
\text { el objetivo de eliminar todos los defectos que } \\
\text { pudieran ser ocasionados por esta causa. }\end{array}$ & \\
\hline 4
\end{tabular}

\section{CONCLUSIÓN}

En el trabajo documentado, se logra demostrar que: el docente asumiendo su papel de mediador del proceso enseñanza aprendizaje y el estudiante siendo protagonista de su propia formación es responsable de conocer los contenidos de aprendizaje y desarrollar la capacidad de resolver problemas reales en el ámbito profesional de una forma íntegra.

Esto se logra a través la combinación de saberes : saber, ser y hacer que, además de una implementación de estrategias didácticas idóneas, para la materia de Calidad Aplicada a la gestión, dado su contenido temático se optó por el desarrollo de prácticas situadas, por lo que fue posible comprobar cómo al desarrollar actividades prácticas los estudiantes son capaces de aplicar sus conocimientos a través de la identificación de oportunidades de mejora tipificadas directamente por ellos mismos, esta habilidad la generan vinculando la teoría con la práctica a través de escenarios reales que los lleven a afrontar retos personales y profesionales y los motiven a seguir desarrollando su saber.

El desarrollo de prácticas situadas en contextos reales en donde la empresa les da la oportunidad de adentrarse en el mundo laboral de una forma directa y asumiendo responsabilidades les permite identificar áreas de oportunidad en dónde puedan demostrar su capacidad para la resolución de problemas y a su vez fortalecer el desarrollo de competencias 
profesionales que les permitan lograr una actualización de conocimientos teóricos adquiridos directamente en la práctica profesional.

Por otra parte, a través de la implementación de proyectos prácticos con enfoque en la solución de problemas se puede identificar las competencias que se deben fortalecer en el estudiante de Ingeniería en Gestión Empresarial para mejorar la calidad de la educación a través de la de combinación de la teoría con la práctica y alentando al desarrollo de investigación.

Específicamente durante el desarrollo de este proyecto se logró la identificación y clasificación de los principales defectos que se presentan las piezas analizadas, así como la determinación de sus principales causas. El uso de las herramientas estadísticas de la calidad le permitió al estudiante residente llevar a cabo las funciones de: recopilación de información, interpretación y análisis, para así dar a conocer los problemas que se presentaban en las actividades realizadas, su interpretación es objetiva y confiable por ello contribuye a la toma de decisiones efectivas por parte de las personas involucradas para mejorar y optimizar todo tipo de recursos en la empresa así mismo mejorar su imagen a través de la satisfacción y fidelización de los clientes

\section{Recomendaciones}

Se recomienda que los docentes asuman y garanticen su rol de docente mediador a través de una correcta identificación de situaciones prácticas que generen en el estudiante una verdadera motivación por su actualización profesional, es importante la vinculación escueladocente-empresa-estudiante en materias que aportan competencias al perfil de egreso del estudiante de Ingeniería en gestión Empresarial finalmente para poder seguir con la colaboración mutua en beneficio de todas las partes interesadas para con ello lograr un beneficio al identificar oportunidades de áreas y proyectos en donde puedan participar los estudiantes y que a su vez se logre un impacto positivo en su formación a su vez que se logra una mejora en la calidad de la educación, se actualiza al docente y se permite el cumplimiento de objetivos, metas e indicadores de ambos sectores.

\section{BIBLIOGRAFÍA}

Acosta González, M. G., \& al, e. (Diciembre de 2012). Modelo Educativo del siglo XXI: Formación y desarrollo de competencias profesionales. (V. Pérez, \& L. Gabriela, Edits.) México, Mexico. Obtenido de http://www.dgest.gob.mx/director-general/modelo-educativo-para-elsiglo-xxi-formacion-y-desarrollo-de-competencias-profesionales-dp2

Cobo Gonzales, G., \& Valdivia Cañotte, S. M. (2017). Aprendizaje basado en proyectos. Obtenido de Colección Materiales de Apoyo a la Docencia \#1 @Pontificia Universidad Católica del Perú: https://idu.pucp.edu.pe/wp-content/uploads/2017/08/5.-Aprendizaje-Basado-enProyectos.pdf

Díaz Barriga Arceo, F. (2006). Enseñanza situada, vínculo entre la escuela y la vida. Mexico: Mc. Graw Hill. Recuperado el 01 de sep de 2021, de https://www.uv.mx/rmipe/files/2016/08/Ensenanza-situada-vinculo-entre-la-escuela-y-lavida.pdf

Díaz Barriga, F. (2003). Cognición situada y estrategias para el aprendizaje significativo. Revista Electrónica de Investigación Educativa., Vol. 5(No. 2). Obtenido de http://redie.ens.uabc.mx/vol5no2/contenido-arceo.htm 
Fajardo Pascagaza, E. \&. (2019). El aprendizaje basado en proyectos y su relación con el desarrollo de competencias asociadas al trabajo colaborativo. Rev. Amauta, Vol. 17( Núm. 33 ).

Obtenido de

http://investigaciones.uniatlantico.edu.co/revistas/index.php/Amauta/article/view/2255

Ferreiro Martínez, V. V., Brito Laredo, J., Isabel Garambullo, A., \& Martínez López, C. (2012).

Proyectos De Vinculación Escuela-Empresa Como Estrategia De Apoyo En La Calidad Del Proceso Enseñanza Aprendizaje De La Educación Superior. Rev. Internacional Administración \& Finanzas, Vol. 5(No. 3). Recuperado el 23 de may de 2021, de https://www.theibfr.com/download/riaf/2012-riaf/riaf-v5n3-2012/RIAF-V5N3-2012-8.pdf

Maldonado Pérez, M. (2008). APRENDIZAJE BASADO EN PROYECTOS COLABORATIVOS. Laurus, Vol. 14 (No. 28), 158-180.

Saeger, A. (2016). El diagrama de Ishikawa, solucionar problemas desde su raiz. 50 minutos.es. 